\title{
Respiratory Failure Following Interscalene Block for the Treatment of Shoulder Dislocation in a Patient with COVID-19
}

\author{
Elham Mousavi (iD ${ }^{1}$ and Masood Mohseni (iD) ${ }^{2, *}$ \\ ${ }^{1}$ Department of Sports Medicine, Iran University of Medical Sciences, Tehran, Iran \\ ${ }^{2}$ Department of Anesthesiology, Iran University of Medical Sciences, Tehran, Iran \\ "Corresponding author: Department of Anesthesiology, Iran University of Medical Sciences, Tehran, Iran. Email: masood.mohseni@gmail.com
}

Received 2021 July 03; Revised 2021 August 01; Accepted 2021 August 01.

\begin{abstract}
Introduction: Guidelines recommend the use of regional anesthesia for patients with COVID-19, when possible. Interscalene block (ISB) is the standard care for analgesia of shoulder surgery. Hemidiaphragmatic paresis due to phrenic nerve block is expected in ISB but is usually well-compensated. We present a patient with pulmonary involvement of COVID-19 candidate for the surgery of shoulder dislocation under ISB who experienced respiratory failure after the block.

Case Presentation: A36-year-old female patient with COVID-19 developed a shoulder dislocation following a seizure. Relocating the joint was successfully attempted under intravenous sedation and ultrasound-guided ISB. The patient developed respiratory distress due to hemidiaphragmatic paresis after the block. She was managed using a continuous positive airway pressure (CPAP) mask for 48 hours until the pulmonary condition improved.

Conclusions: Anesthetists should prepare themselves to replace ISB with diaphragm-sparing blocks or apply techniques to reduce the chance of hemidiaphragmatic paresis after ISB in patients with COVID-19.
\end{abstract}

Keywords: COVID-19, Brachial Plexus Block, Nerve Block, Respiratory Insufficiency

\section{Introduction}

The coronavirus 2019 (COVID-19) has threatened world health with more than 125 million confirmed cases worldwide up to the time of writing this manuscript (1). Recent guidelines suggest that surgery should be delayed for at least seven weeks following COVID-19 infection when possible (2). However, the huge scale of the pandemic means that a significant number of patients during the active or recovery phase of COVID-19 will require surgery. Given the involvement of the respiratory system in these patients, guidelines suggest regional anesthesia techniques whenever possible (3). It prevents residual muscle paralysis following general anesthesia and can preserve respiratory function. Additionally, it avoids aerosolization and airway instrumentation, which is the main source of viral transmission. However, regional anesthesia may not be considered fully safe in COVID-19 patients (4). We present a patient with pulmonary involvement of COVID-19 candidate for shoulder dislocation under regional anesthesia who experienced an exacerbation of pulmonary symptoms following interscalene block (ISB).

\section{Case Presentation}

The patient was a 36-year-old woman hospitalized with symptoms of COVID-19 and a PCR test, confirming the diagnosis admitted to Rasoul Akram University Hospital in June 2020. Her symptoms included fever, myalgia, headache, dizziness, cough, and shortness of breath with oxygen saturation of $85 \%$ on admission. She was being treated with supplemental vitamins, acetaminophen, remdesivir, and dexamethasone. She had a seizure on the second day of hospitalization. On the second day of hospitalization, she suffered a seizure resulting in a dislocated shoulder. X-ray assessment confirmed anterior shoulder dislocation without any fracture.

She was taken to the operating room for close reduction. Her vital signs were blood pressure 134/82 $\mathrm{mmHg}$, temperature $38.2^{\circ} \mathrm{C}$, heart rate 112 , and respiratory rate of 24 with oxygen saturation of $92 \%$ with a simple oxygen face mask. For intravenous anesthesia, low doses of midazolam, fentanyl, and ketamine were used to maintain spontaneous respiration. The first attempt to treat the dislocated shoulder was unsuccessful, and the surgeon requested more muscle relaxation and sedation. It was decided to do regional anesthesia for the patient. Ultrasound- 
guided ISB with posterior in-plane approach with $20 \mathrm{~mL}$ of ropivacaine $0.5 \%$ was performed. The block and the following attempt to relocate the shoulder were successful.

After the procedure, the patient was fully conscious but complained of worsening shortness of breath. The oxygen saturation was unchanged, but some degrees of respiratory distress were evident. A portable chest X-ray was organized to rule out any serious complications due to ISB, such as pneumothorax. It revealed an elevated hemidiaphragm, indicating unilateral diaphragmatic paralysis due to the phrenic nerve block caused by ISB. Owing to the exacerbation of respiratory distress, she was seated upright, and a continuous positive airway pressure (CPAP) mask was applied for her with pressure adjustment, as tolerated. She was transferred to the intensive care unit and continuously monitored for vital signs and oxygen saturation. Respiratory distress was improved somewhat after about 10 hours, but we could not wean the patient from the CPAP mask until about 48 hours after the procedure. During this time, titrated doses of midazolam and dexmedetomidine were administered to sedate the patient. The setting of endotracheal intubation was ready at the patient's bedside, but it was not needed. After 48 hours the CPAP mask was replaced with high-flow oxygen with a non-rebreathing face mask. Respiratory symptoms were improved within eight days. The patient was discharged and transferred to the home quarantine for two weeks.

\section{Discussion}

The most important cause of death in patients with COVID-19 is respiratory failure. In addition to supportive and medical treatments, any procedure that aggravates the patient's respiratory failure should be avoided. When surgery is unavoidable, guidelines suggest regional anesthesia techniques due to their minimal respiratory depression compared with general anesthesia (5). Interscalene block is a known anesthetic technique for shoulder and upper extremity surgeries and postoperative pain control. It results in hemidiaphragmatic paresis in up to $100 \%$ of patients (6). Phrenic nerve involvement in ISB results in a $20 \%-25 \%$ decrease in forced vital capacity (FVC) (7). This decrease in respiratory capacity is well-compensated in healthy patients and is usually asymptomatic. If subjective dyspnea occurs, changing the position to sitting upright, reassuring the patient, and using supplemental oxygen for a few hours is usually sufficient. This is generally transient and known to resolve upon resolution of the anesthetic block. However, in patients prone to respiratory failure, such as in patients with COVID-19, this could be clinically important and even a catastrophe.
Some tricks have been suggested to reduce the chance of post-ISB diaphragmatic paresis. These include ultrasound-guided ISB with small volumes $(5 \mathrm{~mL})$, dilute local anesthetic (LA). These techniques have reduced the chance of paresis to as low as 20\% (7). Ultrasound-guided ISB with the aid of nerve stimulator through extrafascial approach reduces the risk of diaphragmatic paresis and pulmonary impairment when compared with intrafascial approach (8).

It is important to keep in mind that although postISB diaphragmatic paresis is generally transient, several cases of persistent diaphragmatic have been reported ( 9 , 10). Long-term prognosis is thought to be quite good with spontaneous recovery by 6 - 12 months with normalization of vital capacity (11). The possible risk factors for prolonged diaphragmatic paresis include intraneural injection of local anesthetics, direct nerve injury with a needle, neurotoxicity from use of local anesthetics, nerve compression from hematoma, and surgical malpositioning $(12,13)$. Shoulder surgery itself may induce phrenic nerve injury (14). The use of ultrasound in performing blocks reduces the risk of some of these factors.

The efficacy and safety of several diaphragm-sparing nerve blocks have been investigated in recent years. Earlier studies have suggested that supraclavicular block with posterolateral approach to the brachial plexus, C7 root block, anterior suprascapular nerve block, combined axillary-suprascapular nerve blocks, combined infraclavicular-suprascapular blocks, and superior trunk block may provide acceptable postoperative analgesia for shoulder surgery, coupled with a hemidiaphragm paresis incidence of less than $10.7 \%$ (15-17). A retrospective analysis has also suggested that the incidence of hemidiaphragmatic paralysis is significantly lower with costoclavicular (2.5\%) than with supraclavicular brachial plexus block (18). Future randomized trials are required to validate the efficacy of these blocks for shoulder and upper extremity surgery.

In conclusion, anesthetists should prepare themselves to replace ISB with diaphragm-sparing blocks or apply techniques to reduce the chance of hemidiaphragmatic paresis after ISB in patients with COVID-19. Clinicians should also be wary of the higher risk of developing pneumothorax in techniques such as supraclavicular block. After all, we should expect respiratory failure after even regional anesthesia and be prepared for advanced respiratory care.

\section{Footnotes}

Authors' Contribution: Masood Mohseni did conception and critical revision of the manuscript. El- 
ham Mousavi did literature review and drafting of the manuscript.

Conflict of Interests: None to disclose.

Funding/Support: None to disclose.

Informed Consent: Informed consent was obtained from the patient.

\section{References}

1. WHO. WHO Coronavirus (COVID-19) Dashboard. 2021. Available from: https://covid19.who.int/.

2. COVIDSurg Collaborative; GlobalSurg. Timing of surgery following SARS-CoV-2 infection: an international prospective cohort study. Anaesthesia. 2021;76(6):748-58. doi: 10.1111/anae.15458. [PubMed: 33690889]. [PubMed Central: PMC8206995].

3. Lie SA, Wong SW, Wong LT, Wong TGL, Chong SY. Practical considerations for performing regional anesthesia: lessons learned from the COVID-19 pandemic. Can J Anaesth. 2020;67(7):885-92. doi: 10.1007/s12630-020-01637-0. [PubMed: 32212103]. [PubMed Central: PMC7095295].

4. Aminnejad R, Shafiee H. Is Regional Anesthesia Safe Enough in Suspected or Confirmed COVID-19 Patients? ACS Chem Neurosci. 2020;11(9):1371. doi: 10.1021/acschemneuro.0c00146. [PubMed: 32227844].

5. Herman JA, Urits I, Kaye AD, Urman RD, Viswanath O. COVID-19: Recommendations for regional anesthesia. J Clin Anesth. 2020;65:109885. doi: 10.1016/j.jclinane.2020.109885. [PubMed: 32454343]. [PubMed Central: PMC7237915].

6. Urmey WF, Talts $\mathrm{KH}$, Sharrock NE. One hundred percent incidence of hemidiaphragmatic paresis associated with interscalene brachial plexus anesthesia as diagnosed by ultrasonography. Anesth Analg. 1991;72(4):498-503. doi: 10.1213/00000539-199104000-00014. [PubMed: 2006740].

7. Tran DQ, Elgueta MF, Aliste J, Finlayson RJ. Diaphragm-Sparing Nerve Blocks for Shoulder Surgery. Reg Anesth Pain Med. 2017;42(1):32-8. doi: 10.1097/AAP.0000000000000529. [PubMed: 27941477].

8. Ayyanagouda B, Hosalli V, Kaur P, Ambi U, Hulkund SY. Hemidiaphragmatic paresis following extrafascial versus conventional intrafascial approach for interscalene brachial plexus block: A doubleblind randomised, controlled trial. Indian J Anaesth. 2019;63(5):375-
81. doi: 10.4103/ija.IJA_69_19. [PubMed: 31142881]. [PubMed Central: PMC6530291].

9. Robaux S, Bouaziz H, Boisseau N, Raucoules-Aime M, Laxenaire MC, S. O. S. Regional Hot Line Service. Persistent phrenic nerve paralysis following interscalene brachial plexus block. Anesthesiology. 2001;95(6):1519-21. doi: 10.1097/00000542-200112000-00035. [PubMed: 11748414].

10. Deruddre S, Vidal D, Benhamou D. A case of persistent hemidiaphragmatic paralysis following interscalene brachial plexus block. J Clin Anesth. 2006;18(3):238-9. author reply 239. doi: 10.1016/j.jclinane.2005.03.010. [PubMed:16731333].

11. $\mathrm{Xu} \mathrm{WD,} \mathrm{Gu} \mathrm{YD,} \mathrm{Lu} \mathrm{JB,} \mathrm{Yu} \mathrm{C,} \mathrm{Zhang} \mathrm{CG,} \mathrm{Xu} \mathrm{JG.} \mathrm{Pulmonary} \mathrm{func-}$ tion after complete unilateral phrenic nerve transection. J Neurosurg. 2005;103(3):464-7. doi: 10.3171/jns.2005.103.3.0464. [PubMed: 16235678].

12. Lynch NM, Cofield RH, Silbert PL, Hermann RC. Neurologic complications after total shoulder arthroplasty. J Shoulder Elbow Surg. 1996;5(1):53-61. doi: 10.1016/s1058-2746(96)80031-0. [PubMed: 8919443].

13. McCool FD, Tzelepis GE. Dysfunction of the diaphragm. $N$ Engl J Med. 2012;366(10):932-42. doi: 10.1056/NEJMra1007236. [PubMed: 22397655].

14. Boardman N3, Cofield RH. Neurologic complications of shoulder surgery. Clin Orthop Relat Res. 1999;(368):44-53. [PubMed: 10613152].

15. Jason K, Olsen A; Panchamia. Adam, W. Amundson. Unique Phrenic Nerve-Sparing Regional Anesthetic Technique for Pain Management after Shoulder Surgery. Case Rep Anesthesiol. 2017;58(12):1285-96. doi: $10.1155 / 2017 / 1294913$

16. Panchamia JK, Olsen DA, Sanchez-Sotelo J, Amundson AW. Combined Selective Nerve Blockade and Local Infiltration Analgesia in a Total Shoulder Arthroplasty Patient With Chronic Pain and Severe Restrictive Lung Disease: A Case Report. A A Case Rep. 2017;9(12):360-3. doi: 10.1213/XAA.0000000000000617. [PubMed: 28767480].

17. Kim DH, Lin Y, Beathe JC, Liu J, Oxendine JA, Haskins SC, et al. Superior Trunk Block: A Phrenic-sparing Alternative to the Interscalene Block: A Randomized Controlled Trial.Anesthesiology. 2019;131(3):521-33. doi: 10.1097/ALN.0000000000002841. [PubMed: 31283740].

18. Oh C, Noh C, Eom H, Lee S, Park S, Lee S, et al. Costoclavicular brachial plexus block reduces hemidiaphragmatic paralysis more than supraclavicular brachial plexus block: retrospective, propensity score matched cohort study. Korean J Pain. 2020;33(2):144-52. doi: 10.3344/kjp.2020.33.2.144. [PubMed: 32235015]. [PubMed Central: PMC7136300]. 